\title{
Intramedullary neurocytomas in the craniocervical spinal cord: A report of two cases and a literature review
}

\author{
ZHENXING SUN ${ }^{1}$, DAN YUAN ${ }^{2}$, ZHIQIANG CUI ${ }^{1}$, YAXING SUN $^{3 *}$, JUNSHENG YANG $^{4}$, \\ PENGXIANG YAN $^{5^{*}}$ and HUANCONG ZUO ${ }^{1}$ \\ ${ }^{1}$ Department of Neurosurgery, Yuquan Hospital, Medical Center, Tsinghua University, Beijing; ${ }^{2}$ Department of Nephrology, \\ The Luhe Teaching Hospital of The Capital Medical University, Beijing; ${ }^{3}$ Department of Psychiatry, \\ The Second Municipal Hospital of Zaozhuang City, Zaozhuang, Shandong; ${ }^{4}$ Department of Oncology, \\ The Municipal Hospital of Zaozhuang City, Zaozhuang, Shandong; ${ }^{5}$ Department of Neurosurgery, \\ Tiantan Hospital, Capital Medical University, Beijing, P.R. China
}

Received November 21,2013; Accepted September 26, 2014

DOI: 10.3892/ol.2014.2616

\begin{abstract}
Central neurocytoma is a relatively rare tumor of the central nervous system. Young adults are most commonly affected, with a similar incidence in males and females. The tumor is predominantly occurs in the ventricular system of the brain. The tumor is benign and exhibits a good response to surgical resection and radiation therapy. The typical central neurocytoma occurs in the supratentorial ventricular system in young adults. Extraventricular neurocytomas are rare in the spinal cord. In the present study, two cases of craniocervical neurocytomas and the clinical presentation, magnetic resonance imaging observations, pathological features and two-year follow-up results are reported. The first case presents a 26 year old male with an intramedullary mass extending from the medualla oblongata to the $\mathrm{T} 4$ segement of the spine. The second case presents the case of a 48 year old female with an intramedullary mass extending from the oblongata to the $\mathrm{T} 2$ segement of the spine. The two patients underwent subtotal resection of the masses and post-operative radiotherapy was administered for three months. Post-operative magentic resoance imaging revealed no tumor recurrence in the two cases, two years after resection. The relevant literature is also discussed.
\end{abstract}

Correspondence to: $\mathrm{Dr}$ Huancong Zuo, Department of Neurosurgery, Yuquan Hospital, Tsinghua University, 5 Shijingshan Road, Shijingshan, Beijing 100040, P.R. China

E-mail: zhenxingsun5118@163.com

*Contributed equally

Key words: central neurocytoma, extraventricular, craniocervical, literature review

\section{Introduction}

Central neurocytoma is a rare grade II tumor of neuronal origin, according to the World Health Organization staging system (1). Young adults are most commonly affected, with a similar incidence in males and females. The tumors usually occur in the ventricular system of the brain (2). Extraventricular neurocytomas are extremely rare. Since the first case was reported by Hassoun et al in 1982 (3), 271 studies regarding ventricular neurocytomas have been published, while only 64 studies regarding extraventricular neurocytoma have been published. By July 2012, extraventricular neurocytoma had been reported in the cerebrum, including the frontal (4), temporal $(5,6)$, parietal (7) and occipital (7) lobes, the pons (8), the skull base $(9,10)$, the vermis of the cerebellum (11), the cerebellum (12), the sellar region (13), the cauda equina (14), the thalamus (15) and the spinal cord (16-25). To date, 17 cases of neurocytoma involving the spinal cord have been reported; nine cases located in the cervical spinal cord $(16,17,19,21-23,25)$ and eight cases located in the thoracic spinal cord $(18,20,22,23)$. The majority of spinal cord neurocytomas do not recur following complete resection and radiation therapy. In the current study, two cases of intramedullary neurocytomas in the craniocervical spinal cord are reported and the clinical features, radiological observations, histopathological presentation and two-year follow-up results are presented, together with a review of the literature. Written informed consent was obtained from both patients.

\section{Case report}

Case one. A 26-year-old male presented to Yuquan Hospital, Tsinghua University (Beijing, China) with a nine-month history of numbness in the right lower limb and a five-month history of progressive weakness of the left upper limb. The patient's general health was good and no relevant family history was reported. Upon neurological examination, muscle power in the left upper limb was rated as grade 4/5, according to the Medical Research Council scale (26), with decreased pinprick sensation in the sole of the right foot. Myoatrophy 

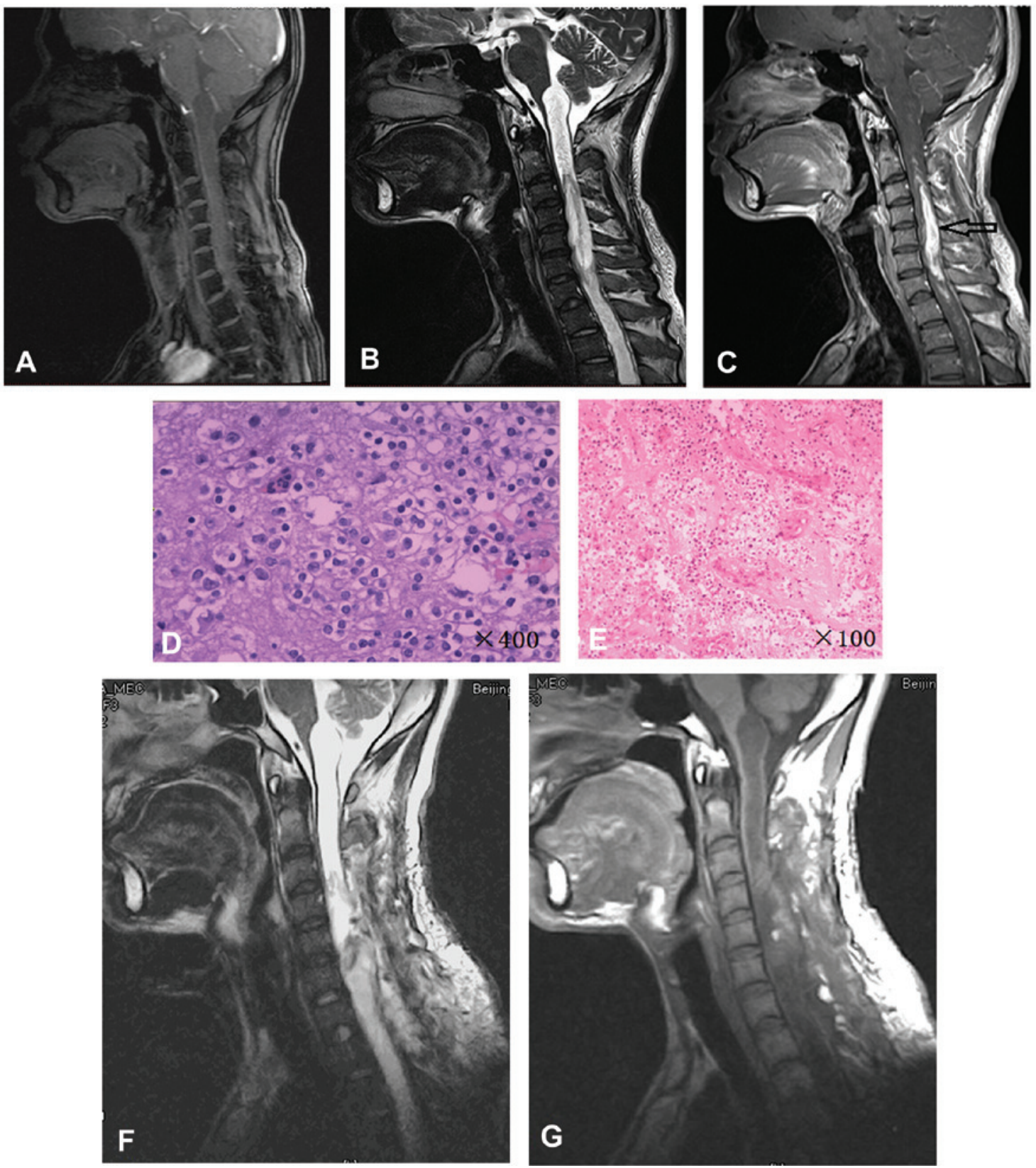

Figure 1. Case one: MRI showing an expansive intramedullary mass extending from the medulla oblongata to the T4 segment of the spine. (A) The mass was isointense on T1-weighted images and (B) hyperintense with a partially cystic mass on T2-weighted images. (C) The mass showed intense heterogeneous enhancement of a solid tumor from the $\mathrm{C} 3$ to $\mathrm{C} 7$ segments following the injection of gadolinium diethylenetriamine pentaacetic acid. (D and E) Hematoxylin and eosin staining from the biopsy of the mass showed a neoplasm composed of uniform, round cells with round, central nuclei and a perinuclear halo [(D), magnification, x400; (E), magnification, x100]. (G and F) Post-operative MRI showing no recurrence of the solid tumor. MRI, magnetic resonance imaging.

was identified in the right lower limb. A physical examination of the spine did not reveal any abnormalities and perineal sensation was normal.

A magnetic resonance imaging (MRI) scan of the craniocervical region revealed an expansile intramedullary mass extending from the medulla oblongata to the $\mathrm{T} 4$ segment of the spine. The mass was isointense on T1-weighted images, hyperintense with partially cystic mass on T2-weighted images and showed intense heterogeneous enhancement of solid tumor following the injection of gadolinium diethylenetriamine pentaacetic acid (Gd-DTPA) (Fig. 1A-C).

A C3-7 laminectomy was performed and an intramedullary solid mass extending from $\mathrm{C} 3$ to $\mathrm{C} 7$ was exposed. The

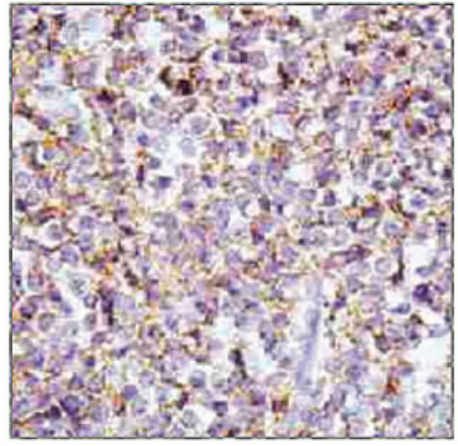

Figure 2. Case one: Immunohistochemical staining revealing positivity for synaptophysin. Magnification, $\mathrm{x} 400$. 

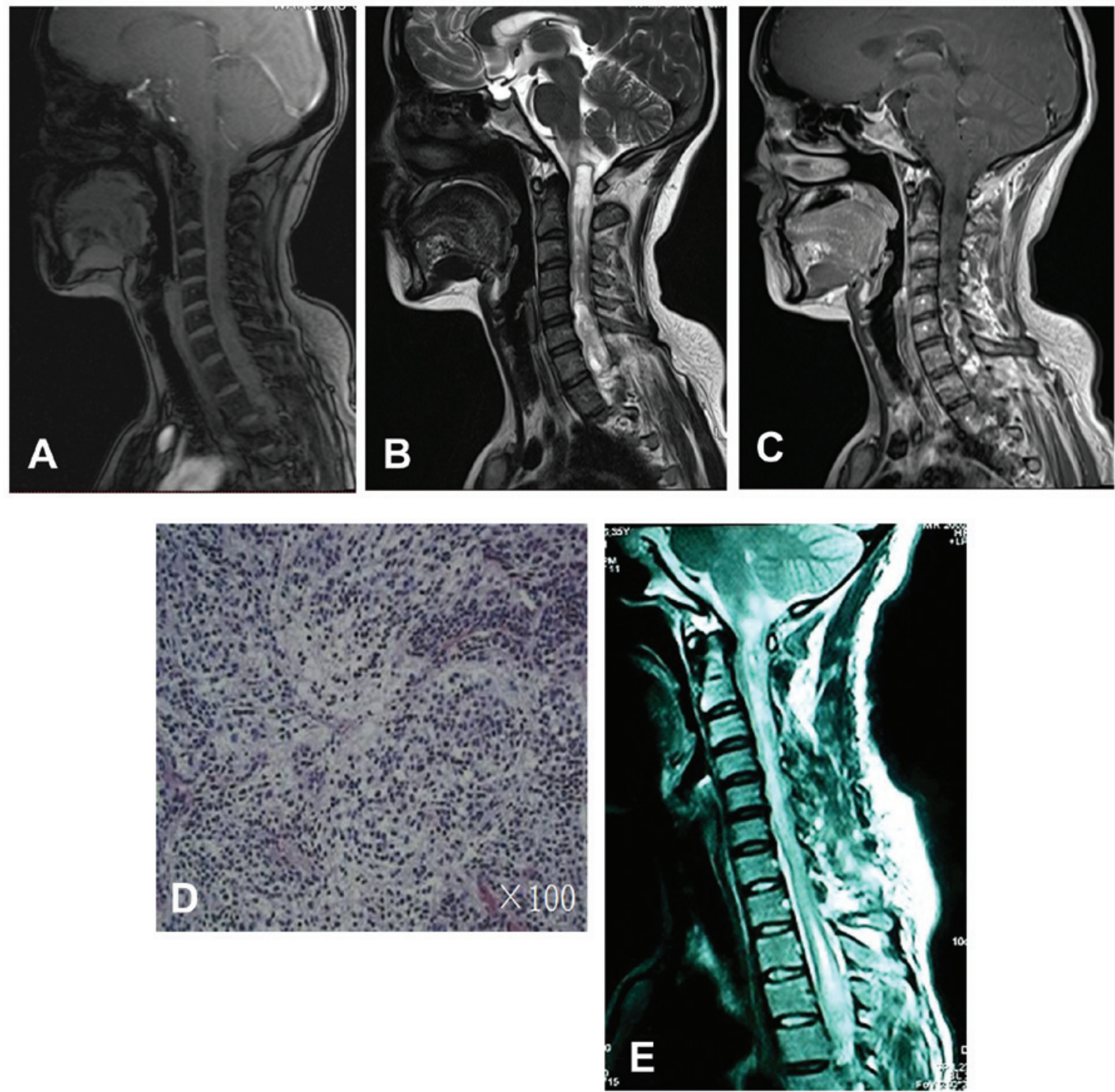

Figure 3. Case two: MRI showing an expansive intramedullary mass extending from the medulla oblongata to the T2 segment. (A) The mass was isointense on T1-weighted images and (B) hyperintense with a partially cystic mass on T2-weighted images. (C) The mass at the C5-T1 level showed intense heterogeneous enhancement of a solid tumor following the injection of gadolinium diethylenetriamine pentaacetic acid. (D) Hematoxylin and eosin staining showed a lesion composed of uniformly small, round cells (magnification, x100). (E) Post-operative MRI showing residual tumor. MRI, magnetic resonance imaging.

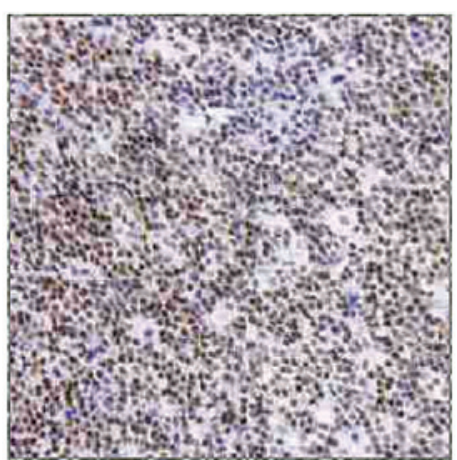

Figure 4. Case two: Immunohistochemical staining revealing positivity for neuronal nuclear antigen. Magnification, x100

tumor was gray-purple and exhibited features of infiltrative growth. A partial tumor resection was performed using a micro-neurosurgery technique, resulting in $85 \%$ of the tumor being resected. Hematoxylin and eosin staining revealed a neoplasm composed of uniform, round cells (Fig. 1D and E). Immunohistochemical staining revealed positivity for glial fibrillary acidic protein (GFAP), neuronal nuclear antigen, vimentin, neuron-specific enolase (NSE), S-100 protein, synaptophysin (SYN; Fig. 2) and oligo2. Post-operative radiotherapy (56 Gy) was administered for three months. Two years after surgery, the patient's symptoms were in remission and post-operative MRI revealed no tumor recurrence (Fig. 1F and G).

Case two. A 48-year-old female presented to Yuaquan Hospital, Tsinghua University, with a seven-year history of pain in the right lower limb and a five-year history of progressive numbness in all four limbs and lumbar zone anesthesia. MRI revealed a mass extending from the medulla oblongata to the T2 level, with an isointense T1-weighted signal and a hyperintense $\mathrm{T} 2$-weighted signal with a partially cystic mass. 
Table I. Summary of the literature review of neurocytomas in the cervical spinal cord.

\begin{tabular}{lccllll}
\hline First author, year (ref.) & $\begin{array}{c}\text { Age, } \\
\text { years }\end{array}$ & Location & $\begin{array}{c}\text { MRI } \\
\text { enhancement }\end{array}$ & Surgery & Radiotherapy & $\begin{array}{c}\text { Recurrence } \\
\text { (follow-up time) }\end{array}$ \\
\hline Tatter et al, 1994 (14) & 65 & C2-C6 & Homogenous & Biopsy & Yes & No (10 years) \\
& 49 & C3-C4 & Homogenous & Total resection & Yes & Yes (30 months) \\
Stapleton et al, 1997 (15) & 12 & C4-T1 & Heterogeneous & Total resection & No & No (24 months) \\
Ashkan et al, 2000 (20) & 12 & C6-T1 & Homogenous & Subtotal resection & No & No (33 months) \\
Sharma et al, 2006 (1) & 24 & C5-T1 & Homogenous & Total resection & No & Yes (10 months) \\
Gokham, 2008 (20) & 49 & C3-C5 & Homogenous & Subtotal resection & No & Unknown \\
Polli et al, 2009 (21) & 15 & C1-T11 & Heterogeneous & Subtotal resection & No & Succumbed \\
& 6 & C1-C7 & Heterogeneous & Subtotal resection & No & No (12 months) \\
Gepp Rde et al, 2012 (23) & 15 & Cervical & Unknown & Subtotal resection & No & Unknown \\
& & spinal cord & & & & \\
\hline
\end{tabular}

MRI, magnetic resonance imaging.

Intense heterogeneous enhancement of a solid tumor was shown following injection of Gd-DTPA (Fig. 3A-C). The tumor capsule could not be clearly observed around the whole of the tumor, so a subtotal resection of the mass was performed. Hematoxylin and eosin staining revealed a lesion composed of uniformly small, round cells (Fig. 3D). Immunohistochemical staining revealed positivity for GFAP, NSE, vimentin and SYN (Fig. 4). Post-operative radiotherapy (56 Gy) was administered for three months and no deterioration was identified. Following radiotherapy, residual tumor was observed by MRI, however, the patient's condition had not deteriorated on a follow-up MRI performed after two years (Fig. 3E).

\section{Discussion}

Neurocytomas are tumors of the central nervous system that are derived from the neuronal precursor cells surrounding the central canal region in the developing fetus (2). The spinal cord is an extremely rare site for extraventricular neurocytomas. To date, the majority of cases of spinal neurocytomas cited in the literature have occurred in the cervico-thoracic region. Neurocytomas occurring in the cervical region of the spinal cord region were first documented in 1994 (16). Since then, only eight cases have been reported in the cervical spinal cord (Table I). In the present study, two cases of neurocytoma occurring in the craniocervical region were reported. These are the first cases to be reported in China.

According to the literature review, six cases of cervical spinal neurocytomas occurred in young adults while three cases were reported in adults. In the current study, one patient was 24 years old and the other was 48 years old. The clinical presentation of neurocytoma depends on the location and size of the tumor. Upon neurological examination of case one, the patient was found to have abnormal sensation and loss of proximal muscle power in the upper limbs. MRI revealed a mass with low to intermediate signal intensity on T1-weighted images and intermediate to high signal intensity on T2-weighted images. These tumors involved multiple spinal segments and contrast-enhanced MRI revealed either homogenous or heterogeneous enhancement of the tumor mass. The tumors did not exhibit any characteristic manifestations and their pre-operative diagnosis was relatively difficult, which is typical of spinal neurocytoma. The differential diagnosis of spinal neurocytomas include ependymoma and oligodendroglioma (19).

Neurocytomas are benign and slow growing, and the majority of patients with ventricular neurocytomas may be treated effectively by surgery. However, total resection of spinal cord neurocytomas is relatively difficult, particularly in tumors involving the upper cervical spinal cord and in those involving multiple segments. Giant cervical cord neurocytomas may be treated with subtotal resection followed by radiotherapy, however, in the present literature review, only the two cases reported by Tatter et al (16) received radiotherapy. By contrast, Stapleton et al (17) reported that post-operative radiotherapy should be avoided, and the remaining six cases did not receive radiotherapy. Of these six cases, one patient succumbed to the tumor and one patient presented with recurrence during follow-up $(19,21-23,25)$. In the present study, for each case, the tumors involved the craniocervical region and were difficult to remove intact. As a consequence, partial and subtotal resections were performed in case one and case two, respectively. Following surgery, the two patients received radiotherapy for three months and no tumor recurrence was observed at the end of the two-year follow-up period. Overall, the number of reported cases of upper cervical neurocytoma is so small that it is unclear whether post-operative radiotherapy is beneficial in preventing tumor recurrence. However, the present study indicates that radiotherapy for a period of three months following surgical resection may prove useful in the prevention of tumor recurrence.

In the present study, the cases of two patients with craniocervical neurocytomas were reported, including the clinical presentation, radiological observations and histopathological 
features. The successful treatment of neurocytomas may be dependent on an early diagnosis and the total surgical resection of the tumors, however, it is unclear whether post-operative radiotherapy is required to prevent tumor recurrence. Although craniocervical neurocytomas are rare and difficult to diagnose, they must be considered as a presurgical differential diagnosis for craniocervical tumors. In addition, a long-term follow-up period is required for craniocervical neurocytomas due to the possibility of local recurrence.

\section{References}

1. Louis DN, Ohgaki H, Wiestler OD, et al: The 2007 WHO classification of tumours of the central nervous system. Acta Neuropathol 114: 97-109, 2007.

2. Sharma MC, Deb P, Sharma S and Sarkar C: Neurocytoma: a comprehensive review. Neurosurg Rev 29: 270-285, 2006.

3. Hassoun J, Gambarelli D, Grisoli F, et al: Central neurocytoma. An electon-microscopic study of two cases. Acta Neuropathol 56: $151-156,1982$.

4. Harada M, Morioka T, Nishio S and Fukui M: Neurocytoma in the left frontal lobe. No Shinkei Geka 19: 89-92, 1991.

5. Treier M, Doostkam S and Meckel S: Extraventricular neurocytoma: a rare cause of temporal lobe epilepsy. Rofo 183: 1065-1066, 2011.

6. Giulioni M, Martinoni M, Rubboli G, et al: Temporo-mesial extraventricular neurocytoma and cortical dysplasia in focal temporal lobe epilepsy. J Clin Neurosci 18: 147-148, 2011.

7. Liebert W, Szymas J, Majewski T and Paprzycki W: Central neurocytoma of the right parietal and occipital lobe, Case report. Neurol Neurochir Pol 32: 191-199, 1998.

8. Swinson BM, Friedman WA and Yachnis AT: Pontine atypical neurocytoma: case report. Neurosurgery 58: E990, 2006.

9. Kowalski RJ, Prayson RA and Lee JH: Skull base neurocytoma: case report and review of the literature of extraventricular neurocytomas. Skull Base 12: 59-65, 2002.

10. Shidoh S, Yoshida K, Saitoh K, et al: Extraaxial neurocytoma in the skull base. Brain Tumor Pathol 28: 273-277, 2011.
11. Kapoor N, Gandhi A and Chaurasia AK: Central neurocytoma in the vermis of the cerebellum. Indian J Pathol Microbiol 52: 108-109, 2009.

12. Ogiwara H, Dubner S, Bigio E and Chandler J: Neurocytoma of the cerebellum. Surg Neurol Int 2: 36, 2011.

13. Wang YY, Kearney T, du Plessis D and Gnanalingham KK: Extraventricular neurocytoma of the sellar region, $\mathrm{Br} \mathrm{J}$ Neurosurg 26: 420-422, 2012.

14. Stephan CL, Kepes JJ, Arnold P, Green KD and Chamberlin F: Neurocytoma of the cauda equina. Case report. J Neurosurg 90: 247-251, 1992

15. Cheung YK: Central neurocytoma occurring in the thalamus: CT and MRI findings. Australas Radiol 40: 182-184, 1996.

16. Tatter SB, Borges LF and Louis DN: Central neurocytomas of the cervical spinal cord. Report of two cases. J Neurosurg 81: 288-293, 1994.

17. Stapleton SR, David KM, Harkness WF and Harding B: Central neurocytoma of the cervical spinal cord. J Neurol Neurosurg Psychiatry 63: 119, 1997.

18. Martin AJ, Sharr MM and Teddy PJ: Neurocytoma of the thoracic spinal cord. Acta Neurochir (Wien) 144: 823-828, 2002.

19. Sharma S, Sarkar C, Gaikwad S, Suri A and Sharma MC Primary neurocytoma of the spinal cord: a case report and review of literature. J Neurooncol 74: 47-52, 2005.

20. Singh A, Chand K, Singh H, Sarkar C and Sharma MC: Atypical neurocytoma of the spinal cord in a young child. Childs Nerv Syst 23: 207-211, 2007.

21. Gokhan GA, Gurer IE and Akyuz: A case of extraventricular neurocytoma of the spinal cord. Neuropathology 28: 322-325, 2008.

22. Ashkan K, Casey AT and D'Arrigo C: Benign central neurocytoma. Cancer 89: 1111-1120, 2000.

23. Polli FM, Salvati M and Miscusi M: Neurocytoma of the spinal cord: report of three cases and review of the literature. Acta Neurochir (Wien) 151: 569-574, 2009.

24. Tsai CY, Tsai TH and Lin CH: Unusual exophytic neurocytoma of thoracic spine mimicking meningioma: a case report and review of the literature. Eur Spine 20 Suppl 2: S239-S242, 2011.

25. Gepp Rde A, Sacco RC and Brandão IC: Central neurocytoma of spinal cord. Arq Neuropsiquiatr 70: 234-235, 2012.

26. Medical Research Council. Aids to the examination of the peripheral nervous system, Memorandum no. 45, Her Majesty's Stationery Office, London, 1981. 